\title{
$\underline{\text { Imaging and fluid biomarkers in frontotemporal dementia }}$
}

Lieke H.H. Meeter, $\mathrm{MD}^{1}$; Laura Donker Kaat, $\mathrm{MD}, \mathrm{PhD}^{1,2}$; Jonathan D. Rohrer, $\mathrm{MD}, \mathrm{PhD}^{3}$; John C. van Swieten, $\mathrm{MD}, \mathrm{PhD}^{1,4}$

${ }^{1}$ Department of Neurology, Erasmus Medical Center, Rotterdam, The Netherlands

${ }^{2}$ Department of Clinical Genetics, Leiden University Medical Center, Leiden, The Netherlands

${ }^{3}$ Dementia Research Centre, Department of Neurodegenerative diseases, Institute of Neurology,

University College London, UK

${ }^{4}$ Department of Clinical Genetics, VU University Medical Center, The Netherlands

Correspondence to: $\quad$ Prof. John C. van Swieten, $\mathrm{MD}, \mathrm{PhD}$

Erasmus Medical Center Rotterdam

Department of Neurology, Room Hs 611

's-Gravendijkwal 230

3015 CE Rotterdam, The Netherlands

Tel: +31 1070332 74, Fax: +31 107044727

E-mail: j.c.vanswieten@erasmusmc.nl

Title: 55/80 characters (incl spaces)

Word count main text: 5684

[Ed: Please fill out a third party rights table

(http://www.nature.com/licenceforms/npg/thirdpartyrights-table.doc) and return to nrclinical@nature.com as soon as possible, so that we can start working on obtaining the permissions to use the previously published figures.] 


\begin{abstract}
Frontotemporal dementia (FTD), the second most common type of presenile dementia, is characterized by progressive behavioral and/or language problems. It is a heterogeneous neurodegenerative disease, and includes a range of clinical, genetic and pathological subtypes. This heterogeneity hampers the diagnostic process, which is becoming increasingly important for future clinical trials on disease-modifying treatments. Reliable biomarkers will enable us to better discriminate FTD from other forms of dementia and predict disease progression in the clinical setting. As different underlying pathologies probably require specific pharmacological interventions, robust biomarkers are essential to select patients with specific FTD subtypes. This review emphasizes the increasing availability and potential applications of imaging (structural and functional) and fluid biomarkers (CSF and blood) in sporadic and genetic FTD. The relevance of new MRI modalities such as VBM, DTI and ASL in the early stages of FTD is discussed, together with their ability to classify FTD subtypes. We highlight promising new fluid biomarkers for staging and monitoring FTD, underlining the importance of large, multicenter studies of presymptomatic FTD subjects. Crucial for the implementation of new biomarkers in clinical practice, is the harmonization in collecting and analyzing across different centers, which will become a great challenge for the next years.
\end{abstract}

\title{
Introduction
}

Frontotemporal dementia (FTD) is the second most common form of dementia in people aged under 65 and encompasses two main clinical manifestations: behavioral changes with executive dysfunction ('behavioral FTD', bvFTD) or predominant language impairment ('primary progressive aphasia', PPA) (Text Box 1). ${ }^{1,2}$ PPAs can be further divided in the semantic variant (svPPA), non-fluent variant (nfvPPA) and logopenic variant (lvPPA). ${ }^{2}$ Patients may develop concomitant parkinsonism or motor neuron disease (MND) at an early or late stage, resulting in a broad clinical phenotype ranging from amyotrophic lateral sclerosis (ALS) to progressive supranuclear palsy (PSP) and corticobasal syndrome (CBS) (Figure 1). ${ }^{3}$ 
Patients presenting with nfvPPA may develop characteristic features of PSP or CBS over time, while lvPPA is frequently associated with underlying Alzheimer's disease (AD).

Postmortem examination of the brain shows frontotemporal lobar degeneration (FTLD) with either tau (FTLD-tau), TAR DNA-binding protein 43 (FTLD-TDP), or fused in sarcoma inclusions (FTLD-FUS). ${ }^{3}$ Correlation between the clinical presentation and specific underlying pathology is poor in bvFTD and better in svPPA and FTD-MND, both associated with TDP pathology. ${ }^{4}$ Patients who develop symptoms consistent with PSP or CBS, often exhibit FTLD-tau at post-mortem examination. In contrast to sporadic FTD, the underlying pathology in genetic FTD can accurately be predicted (Figure 1).

FTD is highly heritable and $10-20 \%$ of all cases are caused by mutations in three genes: microtubuleassociated protein tau $(M A P T)$, progranulin $(G R N)$, and chromosome 9 open reading frame $72(C 9$ orf 72$){ }^{3}$ Other, rare, FTLD genes include charged multivesicular body protein $2 \mathrm{~B}(C H M P 2 B)$, valosin containing protein $(V C P)$, sequestosome 1 (SQSTM1), transactive response DNA-binding protein (TARDP), and the more recently identified TANK-binding kinase 1 gene $(T B K-1) .{ }^{5}$ Although some phenotypes are associated with specific mutations, e.g. the co-occurence of MND with C9orf72 mutations, genotypephenotype correlations are generally poor, even within families. ${ }^{3}$

Sensitive biomarkers are crucial for FTD because of its heterogeneity. Great efforts to identify these biomarkers have been made over the last two decades, with a predominant focus on fluid biomaterial and neuroimaging features. The ideal biomarker should meet the following criteria, according to previous consensus: 1) able to detect a fundamental pathological feature of the disease, 2) validated in pathological proven cohorts, 3) precise, 4) reliable, 5) non-invasive, 6) simple to perform, and 7) inexpensive (Text Box 2). ${ }^{6}$ Different biomarkers can be used for specific purposes (Text Box 2), and therefore the value of a biomarker depends on its application. In FTD, diagnostic biomarkers should discriminate FTD from controls and other neurodegenerative diseases, or differentiate between clinical, genetic or pathologic subtypes. Staging biomarkers should allow us to assess disease severity and to discriminate between 
presymptomatic, prodromal, and early or late symptomatic stages. Pharmacodynamic biomarkers are important for evaluating the biological and clinical effect of future therapeutic interventions. Predicting the underlying pathology in FTD (tau versus TDP-43) is one of the greatest challenges, as this will be essential when specific disease-modifying interventions become available. Ideally, these interventions should be applied at an early stage when only minimal neuronal damage is present, underpinning the need for early biomarkers; at-risk subjects from families with genetic FTD form the ideal study population for detecting these earliest changes.

In this review we focus on fluid and neuroimaging biomarkers in FTD. We discuss previous work on biomarkers with its current application in clinical practice and we highlight the development of new, promising biomarkers.

\section{Neuroimaging biomarkers}

Most FTD imaging studies have focused on structural changes by assessing grey matter atrophy, while more recent studies have been directed towards studying white matter integrity using diffusion tensor imaging (DTI), which is probably more sensitive for the earliest changes in FTD. In neurodegenerative diseases in general, structural abnormalities are often preceded by functional changes, and in the following section, we describe both the structural and functional changes found with different imaging modalities.

\section{Structural changes}

\section{Grey matter}

The majority of imaging studies in FTD have used volumetric T1 MRI to investigate changes in grey matter structure..$^{7-10}$ This technique is used to measure brain volume (and longitudinally, changes in that volume i.e. the rate of brain atrophy) as well as the volume of specific regions of interest within the brain, for example the frontal lobe or hippocampus. Several post-processing analytic techniques have also been 
applied to T1 imaging e.g. investigation of changes at the voxel level (e.g. voxel-based morphometry) or measurement of cortical thickness (e.g. Freesurfer), each providing an alternative way of investigating grey matter loss in the brain.

On an individual patient level, semi-quantitative assessment of atrophy by visual rating scales performed by experienced dementia experts, has provided a good diagnostic performance to discriminate FTD from $\mathrm{AD}$ (where more posterior cortical involvement is seen) with a specificity of $81 \% .^{11}$

Also clinical, genetic and pathological syndromes of FTD can to some degree be distinguished by distinct and dissociable patterns of grey matter atrophy at group level (Figure 2). Clinically, bvFTD is associated with frontal, temporal, insula and anterior cingulate atrophy, with earliest involvement of frontal paralimbic cortices and insula in mild bvFTD. ${ }^{12-14}$ Cluster analyses suggest that there may be four anatomical forms of bvFTD with frontal-dominant, temporal-dominant, frontotemporal and distributed temporofrontoparietal subtypes. ${ }^{15,16}$ However, these analyses have underplayed the involvement of subcortical structures in bvFTD, and it is clear that as the disease progresses, atrophy of the hippocampus, amygdala, basal ganglia and thalamus occurs. ${ }^{14,17}$ In the PPA syndromes, svPPA is associated with (commonly left-sided) asymmetrical anteroinferior temporal lobe atrophy, nfvPPA with left-sided predominant inferior frontal and insula involvement, and lvPPA with left temporo-parietal junction loss. ${ }^{18,19}$ Over time the extent of atrophy progresses not only within the same hemisphere but also starts to involve the opposite hemisphere in each of the PPA syndromes. ${ }^{20-22}$ In the genetic forms of FTD, GRN mutations are associated with asymmetrical fronto-temporo-parietal atrophy, $M A P T$ mutations with relatively symmetrical involvement of the anteromedial temporal and orbitofrontal lobes, and $C 9$ orf 72 expansions with a symmetrical more widespread pattern of atrophy together with involvement of the thalamus and superior cerebellum. ${ }^{16,23-26}$ As with the clinical and genetic subtypes, whilst there are grouplevel patterns, it has been difficult to distinguish individuals with specific pathological forms of FTD purely using structural T1 imaging, nor has it been possible to distinguish those with FTLD-TDP from 
FTLD-tau. ${ }^{9}$ Patients with FUS pathology generally present with prominent caudate atrophy, accompanied by orbitofrontal, anteriomedial temporal, anterior cingulate, and insula atrophy. ${ }^{27,28}$

Across clinical, genetic and pathological forms of FTD, less work has been done on longitudinal changes in grey matter loss. However it is clear that there are variable rates of atrophy in different groups, with some being relatively fast (e.g. those with $G R N$ mutations), and some very slow (a subgroup of patients with $C 9 \mathrm{orf} 72$ repeat expansions).${ }^{29}$ If longitudinal structural imaging would be used for monitoring in clinical trials, sample size estimations show that focal atrophy rates, e.g. temporal lobe in svPPA, would allow a smaller sample than whole brain atrophy rates (reviewed elsewhere). ${ }^{7}$

Findings from a number of small studies of those at-risk for genetic FTD have been inconsistent, with some showing grey matter atrophy prior to onset of symptoms and others not. However, a recent large multicenter analysis from the GENFI study reported the presence of atrophy at least 10 years prior to expected symptom onset (Figure 3a), with different genetic groups showing different patterns: in MAPT mutations, atrophy was noted first in the hippocampus and amygdala, followed by the temporal lobe and later the insula; in GRN carriers differences started in the insula, followed by temporal and parietal lobes and thereafter the striatum; in the C9orf72 group, changes were found very early ( 25 years before expected onset) in the subcortical areas (including thalamus), insula and occipital cortex, then the frontal and temporal lobes and later the cerebellum. ${ }^{30}$ Prominent asymmetry was found in GRN mutation carriers at five years before expected onset, but not in the other genetic subgroups. It is important to look at changes over time in this cohort, as small-scale longitudinal studies have shown more sensitivity, as illustrated by a significant reduction of left temporal cortical thickness in presymptomatic $G R N$ carriers, without differences between presymptomatic- and non-carriers at baseline. ${ }^{31}$

\section{Diffusion tensor imaging}

DTI is a valuable non-invasive imaging technique for assessing white matter structure of the brain. It measures white matter microstructural integrity by determining the rate of diffusion (motion of water 
molecules) in different directions. Specific DTI metrics are thought to reflect different pathological changes in microstructure: a decrease in axial diffusivity $(\mathrm{AxD})$ correlates with axonal degeneration; an increase in radial diffusivity (RD) indicates myelin breakdown; and a decrease of fractional anisotropy (FA), as a composite measure of both $\mathrm{AxD}$ and $\mathrm{RD}$, represents more general nonspecific white matter integrity loss. ${ }^{32}$ Abnormalities in white matter diffusivity have been found to be more widespread and to grey matter atrophy in FTD, supporting the importance of white matter involvement in FTD. ${ }^{33-38}$ DTI may become a valuable biomarker as it has at least four potential applications, although currently, it has only been investigated at group level and not at single-subject level.

Firstly, DTI is highly sensitive to differentiate FTD from controls and from other types of dementia, such as $\mathrm{AD} .{ }^{32-36,38-45}$ White matter microstructure has shown to be more widespread affected in FTD compared with $\mathrm{AD},{ }^{32,34,39,40}$ with a high sensitivity $(78 \%)$ and moderate specificity $(68 \%)$ to discriminate these conditions by whole brain mean FA. ${ }^{34}$ White matter degradation co-occurs with frontal, temporal and insular atrophy in FTD, and is probably due to axonal degeneration associated with grey matter neuronal loss. It includes the anterior corpus callosum, bilateral anterior and descending cingulum and uncinate fasciculus tracts, ${ }^{42}$ which are part of motor, executive and language neural networks.

Secondly, although white matter damage on DTI largely overlaps between subtypes, some distinctive DTI changes have been found in clinical, pathological and genetic FTD subtypes. $33,35,36,38,40,42-44,46$ The uncinate fasciculus, cingulum bundle and genu of the corpus callosum appear to be key tracts involved in the bvFTD disease process. ${ }^{34,41,44}$ PPA subtypes have shown different spatial patterns of white matter damage: left orbitofrontal and anterior temporal white matter (superior longitudinal fasciculus) in nfvPPA; asymmetric (mostly left) changes in the anterior and inferior temporal white matter (including the inferior longitudinal fasciculus), and bilateral uncinate fasciculi in svPPA; and more posterior abnormalities such as the posterior region of the left inferior longitudinal fasciculus in lvPPA. $33,35,36,38,42-44$ DTI may have the potential to differentiate FTLD-tau from FTLD-TDP in vivo, as two studies have found more severe white matter integrity loss in FTLD-tau than FTLP-TDP. ${ }^{33,46}$ This parallels postmortem 
findings where tau-pathology is associated with marked axonal loss and glial tau-inclusions, and TDPpathology with greater grey matter neuronal loss than white matter pathology. ${ }^{46}$ Larger studies are needed for more conclusive observations before this can be used in individual patients. DTI studies have shown different patterns across genetic FTD patients subtypes: MAPT patients show consistent alterations in the uncinate fasciculus and right parahippocampal cingulum, ${ }^{34,41}$ whereas $C 9$ orf72-FTD patients tend to have more dorsal white matter tract pathology located in the cingulum, corpus callosum and the superior cerebellar peduncles..$^{34,41}$

Thirdly, though studies so far are limited, longitudinal DTI changes may be used to monitor the disease process and evaluate therapeutic effects in future clinical trials. ${ }^{41}$ Over time, DTI changes have been found to be more widespread than progression of grey matter atrophy, and have shown distinct patterns between clinical and genetic FTD subtypes which reflect different propagation of the neurodegenerative process within large scale brain networks. ${ }^{35}$ The bilateral uncinate fasciculus and paracallosal cingulum have shown the largest FA reduction in bvFTD, ${ }^{35,41}$ while left-to-right sided progression is seen in both svPPA and nfvPPA. ${ }^{35,43}$ In svPPA, longitudinal DTI changes extended to bilateral frontotemporal tracts, whereas changes in nfvPPA appeared to remain relatively focal..$^{35,43}$

Lastly, DTI as a biomarker may even be able to detect pathological changes before the onset of clinical symptoms and before grey matter atrophy in FTD. Decreased FA and increased RD in bilateral uncinate fasciculi (and forceps minor) have been found in a group of presymptomatic MAPT or GRN mutation carriers without grey matter atrophy (Figure 3b). ${ }^{37,47}$

In conclusion, DTI appears to be a promising imaging biomarker for early diagnosis, and possibly to monitor the effect of pharmacological interventions in the future. To use DTI in the individual patient, reference data are essential to identify abnormal changes in white matter integrity, alike the use of automated quantitative MRI. ${ }^{48}$ The assembly of such normative data is however challenging due to variability across scanners and field strengths, as well as choice of DTI metric, tract, and method of 
analyzing (e.g. tracking or skeletonized). Region of interest analyses of specific tracts, such as the uncinate fasciculus, inferior - and superior longitudinal fasciculus, are likely to provide the best opportunity to move forward from the current group level studies to a single-subject analysis.

\section{Functional changes}

\section{FDG-PET}

Positron emission tomography with $\left[{ }^{18} \mathrm{~F}\right]$ fluorodeoxyglucose as tracer (FDG-PET) allows the visualization of alterations in brain metabolism, which precede grey matter atrophy in FTD and different forms of dementia. ${ }^{49-52}$ Distinct regional hypometabolism patterns on FDG-PET contribute to an accurate clinical diagnosis at an individual patient level, both by visual inspection and especially by quantitative assessment. ${ }^{53}$ Lower glucose metabolism, often asymmetric, in the orbitofrontal, dorsolateral and medial prefrontal cortex, anterior temporal poles and basal ganglia, is highly specific for bvFTD, with a sensitivity and specificity ranging between 80 and $95 \%$ for the differentiation from other dementia types and healthy controls..$^{50,51,53-56}$ These patterns of hypometabolism are early features in the symptomatic stage, but also a few years before patients fulfill the criteria for probable bvFTD. ${ }^{50}$ As false-positive FDGPETs have been found in primary psychiatric disorders mimicking FTD, future quantitative assessment of metabolism patterns on PET may further increase its diagnostic value. ${ }^{54}$

The patterns of focal hypometabolism vary between PPA subtypes and between genetic forms, and mirror the structural changes described above. svPPA is characteristically associated with asymmetrical bilateral temporal hypometabolism, while nfvPPA shows larger variability in hypometabolic patterns of the left inferior frontal gyrus, dorsolateral frontal cortex, anterior cingulate cortex, insula, and, occasionally, of the parietal cortex. ${ }^{57}$ Distinct FDG-PET patterns in PPAs may predict progression to specific dementia subtypes: bilateral temporo-parietal hypometabolism predicted conversion to $\mathrm{AD}$, parietal hypometabolism to CBS, and involvement of basal ganglia, midbrain and cerebellum to PSP. ${ }^{57}$ Longitudinal changes on FDG-PET may provide additional information on the patterns and speed of 
pathological spread. ${ }^{31,56}$ For example, svPPA patients show bilateral reduction of glucose metabolism in temporal lobes over time, which further extends to the anterior cingulate cortex and the posterior temporal lobes. ${ }^{58}$ When looking at genetic subtypes, $G R N$ mutations are associated with asymmetric hypometabolism in frontal and temporal brain regions, ${ }^{31,59}$ ALS and/or FTD patients with C9orf72 expansions with hypometabolism in the limbic system, basal ganglia and thalamus, ${ }^{60}$ and $M A P T$ mutations with hypometabolism in the medial temporal lobe, frontal and parietal cortices. ${ }^{24}$

Interestingly, FDG-PET already reveals abnormalities in the presymptomatic stage, and may serve as a surrogate endpoint in future therapeutic trials; asymmetric hypometabolism was found in frontal and temporal lobes in asymptomatic GRN carriers preceding the onset of clinical symptoms and of grey matter atrophy. ${ }^{31,59}$

\section{Arterial spin labeling}

Arterial spin labeling MRI (ASL) measures brain perfusion non-invasively by magnetically labeling water protons in arterial blood, which creates an endogenous tracer of cerebral blood flow (CBF). ${ }^{61}$ Brain perfusion measured by ASL correlates very well with metabolism measured by FDG-PET, ${ }^{51,53}$ but has several advantages over FDG-PET: it can be combined with other MRI techniques in a single session, it is non-invasive, has no radiation exposure, is widely available and is less costly. ${ }^{62}$

ASL has shown hypoperfusion in the insula, the amygdala and several parts of the medial frontal lobes, including the anterior cingulate, in FTD patients. ${ }^{51,53,63-65}$ It differentiated bvFTD from AD at an early phase, with areas under the curve of up to 0.87 for CBF in specific frontal or parietal regions. ${ }^{51,53,63}$ In two comparative studies, the regions of hypoperfusion of ASL-MRI largely overlapped with those of hypometabolism on FDG-PET scans, ${ }^{51,53}$ and diagnostic performance when distinguishing bvFTD from $\mathrm{AD}$ and controls, was similar for both modalities. ${ }^{53}$

ASL may also be an early biomarker in the preclinical stage of genetic FTD. Presymptomatic GRN and MAPT mutation carriers have showed significantly stronger CBF decrease over time than controls (Figure 
3c), independent of grey matter atrophy, in widespread frontal, temporal, parietal, and subcortical regions, with the strongest perfusion decline in subjects who converted to the disease stage. ${ }^{62}$ Some regional ASL changes may be specific for particular gene defects, as hypoperfusion may extend into posterior temporal and parietal regions in $G R N$ mutation carriers. ${ }^{62}$

\section{Resting-state fMRI}

Resting-state functional MRI (RS-fMRI) is a potential biomarker for early diagnosis and disease staging as it measures intrinsic functional connectivity between brain regions on MRI, which can be detected as synchronous patterns of spontaneous low frequency fluctuations in blood-oxygen-level-dependent signals. RS-fMRI is a safe, non-invasive, and repeatable tool, which is sensitive to detecting changes in brain functional connectivity before the onset of clinical symptoms or atrophy on group-level. ${ }^{37,66,67}$ Decreased connectivity between the frontoinsula and anterior cingulate cortex, as part of the salience network, is the most consistent finding in FTD,${ }^{67-71}$ while other studies found normal or increased connectivity. ${ }^{72-74}$ Inconsistent differences (both increased and decreased connectivity) were found for the default mode network in FTD. ${ }^{67-69}$ The discrepancies in functional connectivity may partly be explained by cohort and scanner differences and the wide variation in analytical methods, such as independent component analyses, seed- or region-of-interest approaches, or regional homogeneity analyses. ${ }^{66,68,72,74,75}$

Specific network alterations are also found between clinical and genetic subtypes of FTD. Reduced left temporal lobe connectivity is found in SvPPA, ${ }^{76,77}$ attenuated connectivity in both salience and sensorimotor networks in C9orf72 bvFTD-patients, ${ }^{26}$ and reduced left frontal connectivity in GRN mutations. ${ }^{78}$ In the presymptomatic phase, RS-fMRI may be sensitive to detecting connectivity differences, as altered (both reduced and increased) frontoinsula and/or ACC connectivity have been reported in presymptomatic mutation carriers. ${ }^{37,66,78,79}$

\section{Amyloid and Tau PET tracers}


Several other tracers may serve as diagnostic biomarkers in the differential diagnosis of FTD and AD, and between different pathological subtypes of FTD. PET with an amyloid tracer such as Pittsburgh compound $\mathrm{B}(\mathrm{PiB})$ tracer is a robust and sensitive biomarker for detecting amyloid- $\beta$ deposits, indicating AD pathology, in vivo, ${ }^{80}$ and bvFTD, svPPA and nfvPPA are mostly PiB-negative. Most lvPPA cases represent atypical $\mathrm{AD}$ with a $\mathrm{PiB}$ binding pattern similar to that of $\mathrm{AD},{ }^{81-83}$ while $1 \mathrm{vPPA}$ with negative PiB-PET is accompanied by structural and FDG-PET abnormalities supporting underlying FTLD pathology. ${ }^{83,84}$ Unexpected positive PiB-PET in FTD cases may result from mild co-incidental AD pathology, not related to the clinical FTD presentation. ${ }^{85}$

Several tracers have been developed to visualize tau pathology in vivo however the ideal ligand that captures the wide range of tau pathology has not yet been developed. Distinct ligand selectivity to the different tau isoforms and their intracellular aggregation requires probably the application of different tau ligands. ${ }^{86}$ Tau PET with the ${ }^{18} \mathrm{~F}-\mathrm{AV}-1451$ ligand has shown increased uptake in the temporal cortex, frontal cortex, and basal ganglia in FTD patients with an R406W MAPT mutation, which is associated with both 3-repeat- and 4-repeat-tau pathology. In these patients, higher regional ${ }^{18} \mathrm{~F}-\mathrm{AV}-1451$ uptake correlated with lower glucose metabolism and with postmortem burden of tau pathology. ${ }^{87}$ However, binding of ${ }^{18} \mathrm{~F}-\mathrm{AV}-1451$ appears poorer in conditions with only 4-repeat-tau, as shown by a lack of correlation with postmortem tau pathology in PSP. ${ }^{88,89}$ A recent study using postmortem material reported that ${ }^{11} \mathrm{C}-\mathrm{PBB} 3$ was more robust for capturing wide-range tau pathologies, including both 3- and 4-repeatconditions. ${ }^{90}$ Also the ${ }^{18} \mathrm{~F}-\mathrm{THK}-5351$ ligand showed promising results in the 4-repeat-diseases PSP and CBS both on postmortem tissue as in vivo. ${ }^{91,92}$ Once validated, tau PET may become effective in diagnosing underlying tau pathology as well as providing a surrogate marker for trials with anti-tau therapeutics. ${ }^{86}$

\section{$\underline{\text { Summary of imaging biomarkers }}$}


Grey matter atrophy and FDG-PET hypometabolism are validated diagnostic biomarkers showing relatively consistent changes at group level between studies, and are clinically applied at an individual level for the differentiation between FTD and AD or controls (Table 1). More work needs to be done on the use of imaging modalities in distinguishing FTD subtypes at an individual level, with larger studies of longitudinally acquired imaging data, before this can be used as an outcome measure to monitor disease progression in clinical trials.

We expect new modalities like DTI, ASL and RS-fMRI to become valuable biomarkers in clinical practice, especially due to their sensitivity, enabling early diagnosis and their potential use in longitudinal monitoring (Table 1). Crucial to their implementation is the harmonization across different centers, as there is considerable variation across scanners and protocols. For example in ASL, the diversity of scanning protocols influences perfusion quantification, which may be overcome by proposed international standardization of protocols. ${ }^{61}$ Additionally, integrating different types of information by combining imaging modalities holds great promise for the future, as demonstrated by multimodal analyses that have improved the classification between FTD and AD, ${ }^{40,45,52,65,93,94}$ and between clinical FTD subtypes. ${ }^{94}$

\section{Fluid biomarkers}

Alterations in specific protein concentrations in different human fluid compartments may reflect pathophysiological changes of disease processes. The close vicinity of CSF to the brain offers a high chance of revealing disease-specific biomarkers. Subsequent validation in blood of such biomarkers would be of great value, being minimally invasive and enabling repeated measurements over time. Recent studies have demonstrated that brain specific proteins in neurodegenerative disorders can reliable be detected in blood by novel ultrasensitive assays (e.g. Single Molecule Array technology). The progress of these developments in the following years, offers a new window of opportunities for diagnosing, staging and monitoring FTD patients. In the next section, we will first review the currently applied CSF 
markers for differentiating FTD from AD, and then highlight promising (also blood-derived) biomarkers in sporadic and genetic FTD.

\section{CSF amyloid- $\beta$ and tau}

The core CSF biomarkers for AD are phospho-tau 181 (p-tau), total-tau (t-tau), and amyloid beta ${ }_{1-42}\left(\mathrm{~A} \beta_{1-}\right.$ 42); these represent the pathological changes in $\mathrm{AD}$, being accumulation of hyperphosphorylated tau in neurofibrillary tangles, neuronal loss, and amyloid beta (A $\beta$ ) depositions in senile plaques respectively. ${ }^{95}$ These biomarkers have comprehensively been validated to exclude AD in the diagnostic work-up of FTD, both in clinical cohorts and small pathologically confirmed case series, with higher p-tau and t-tau, and lower $A \beta_{1-42}$ levels in $\mathrm{AD}$ than FTD patients. ${ }^{96} \mathrm{~A}$ high $\mathrm{A} \beta_{1-42}: \mathrm{p}$-tau or $\mathrm{A} \beta_{1-42}$ :t-tau ratio gives an especially good diagnostic performance when distinguishing FTD from $\mathrm{AD}\left(\mathrm{A} \beta_{1-42}: \mathrm{p}\right.$-tau: specificity $80 \%$ and sensitivity $87 \%$; $A \beta_{1-42}:$ t-tau: specificity $79 \%$, sensitivity $89 \%$ ). The use of ratios of other $A \beta$ isoforms may improve diagnostic accuracy, especially when differentiating AD from vascular dementia and LBD, but also for distinguishing AD from FTD. ${ }^{97,98}$

The core $\mathrm{AD}$ biomarkers are also valuable when differentiating between underlying AD or FTLD pathology in the differential diagnosis of PPA, in which an AD profile is often found in clinically diagnosed lvPPA patients as opposed to svPPA and nfvPPA patients. ${ }^{99-102}$ An AD CSF profile occasionally occurs in FTD cases, even in pathology proven cases; this may partly be explained by the cooccurrence of $\mathrm{AD}$ pathology. ${ }^{103}$ Moreover, decreased $\mathrm{A} \beta_{1-42}$ levels were found in up to $25 \%$ C9orf72 patients in a Finish cohort, but not in $G R N$ patients, and to elucidate its pathophysiological significance, more clinicopathological and genetic studies are required. ${ }^{104-106}$

Tau levels in CSF are not increased in FTD with underlying tau-pathology nor in patients with MAPT mutations, compared to tau-negative or sporadic FTD. ${ }^{107,108}$ Reduced p-tau:t-tau ratios have been found to be a specific biomarker to distinguish FTLD-TDP from FTLD-tau, although this appears to be driven by concomitant MND. ${ }^{109-112}$ Whether the increase of t-tau as abnormal axonal biomarker or reduction of p- 
tau determines the reduced ratio, is not completely clear. In line with this hypothesis of axonal damage, the association of a reduced p-tau:t-tau ratio with survival is an interesting finding in one study. ${ }^{111}$

\section{Neurofilament proteins}

Neurofilament light chain (NfL) is probably the most promising fluid biomarker in FTD in the short term for disease monitoring and prognosis. Neurofilaments are the major constituent of the neuroaxonal cytoskeleton and play important roles in axonal transport and in the synaps. ${ }^{113} \mathrm{NfL}$ is the most abundant and soluble neurofilament-subunit, and increased levels are thought to reflect axonal damage.

NfL levels are 2.5-11 times higher in FTD than in controls and its clinical value especially lies in the correlation with disease severity and progression, survival, and cerebral atrophy (Figure 4). ${ }^{114-116,111,117-119}$ CSF NfL is also increased, although to a lesser extent, in several other neurodegenerative diseases (e.g. ALS, AD, PSP, and vascular dementia), and should therefore be combined with disease specific biomarkers. ${ }^{14,117,120-122}$ NfL levels are equally elevated among FTD subtypes bvFTD, nfvPPA and svPPA, and are strongly increased in FTD with MND. ${ }^{11,114-116,118}$ High CSF NfL levels were found in patients with TDP pathology compared with tau pathology, which was largely driven by ALS co-occurrence. ${ }^{111,118}$ In particular, high NfL levels were found in FTD patients with GRN mutations, a large variation was found in C9orf72 patients (ranging from high levels in concomitant MND to low levels in patients who slowly progress), and MAPT patients had relatively low levels (Figure 4b). ${ }^{115}$ Interestingly, presymptomatic genetic FTD carriers show normal NfL levels in CSF and blood, with a sharp increase reported after conversion to the disease stage in two converters (Figure 4b). ${ }^{115}$ It is unknown whether and how NfL levels fluctuate over time in FTD, but available longitudinal data in ALS have shown stable NfL levels or a minor increase over time. ${ }^{120,123}$ The recent finding of the strong correlation of NfL levels between CSF and serum makes this biomarker measurable in blood and especially suitable for repeated measurements. ${ }^{115,119}$ 
In mouse models of neurodegenerative diseases (tau, $A \beta$, and $\alpha$-synuclein), NfL increase coincided with the onset and progression of brain pathology, and blocking A $\beta$ lesions attenuated the NfL increase. ${ }^{124}$ This observation suggests that we can use NfL to monitor treatment response in neurodegenerative diseases. In conclusion, NfL is a promising, non-invasive biomarker for disease staging, monitoring, and prognosis in FTD. Longitudinal studies in FTD need to be conducted to gain insights into the role of NfL as a progression marker.

\section{$\underline{\text { Genetic-specific biomarkers }}$}

\section{Progranulin}

Progranulin (PGRN), a multifunctional protein, plays an important role in neurite outgrowth and inflammation. ${ }^{125}$ Due to haploinsufficiency, pathogenic loss of function mutations in $G R N$ reduce blood and CSF PGRN to $25-40 \%$ of normal levels. ${ }^{125-129}$ Blood or CSF PGRN are diagnostic biomarkers for pathogenic GRN mutations, as they discriminate (presymptomatic and symptomatic) carriers from noncarriers with a sensitivity and specificity of up to $100 \%$ (Figure 5a). ${ }^{128,129}$ In line with this, blood PGRN levels can help to assess the pathogenicity for unclassified variants in GRN. Currently, therapeutic trials are focusing on enhancing PGRN expression, for example by histone deacetylase inhibitors. ${ }^{130}$ In these trials, target engagement is assessed using blood PGRN levels, as they appear to be constant over time. ${ }^{129,131}$ However, blood and CSF PGRN are differentially regulated, as demonstrated by the moderate correlation between these compartments in GRN mutation carriers, and therefore CSF should be sampled as well. ${ }^{129}$ PGRN levels thus provide a good pharmacodynamic biomarker, but do not reflect the extent of neurodegeneration in the brain, wherefore different additional biomarkers are needed as surrogate endpoints.

\section{Dipeptide-repeats in C9orf72}


C9orf72 repeat expansions are known to be transcribed to expanded $\mathrm{G}_{4} \mathrm{C}_{2} \mathrm{RNA}$, which forms RNA foci and in parallel is translated into proteins of repeating dipeptides (dipeptide-repeats, DPRs) by repeatassociated non-ATG translation. ${ }^{132}$ Both RNA foci and DPRs are believed to have a key role in the pathophysiology of this disorder. ${ }^{132-134}$ An elevated poly(GP) level, one of the DPRs, has been found in the CSF of patients with C9orf72 repeat expansions, and also in presymptomatic mutation carriers (Figure $5 b+c) .{ }^{134,135}$ Moreover, poly(GP) levels remained relatively constant over time, which support the use of poly $(\mathrm{GP})$ as a potential pharmacodynamic biomarker in future therapeutic trials. ${ }^{135}$ Antisense oligonucleotides binding to $\mathrm{G}_{4} \mathrm{C}_{2}$ RNA have been shown to reduce extracellular poly(GP) in human cell models and to reduce RNA foci and DPRs, as well as CSF poly(GP) levels, in mice harboring a $\mathrm{G}_{4} \mathrm{C}_{2}$ expansion. ${ }^{133-135}$ This implicates that poly(GP) may be a potential target engagement biomarkers to measure biochemical responses to treatment with these agents. ${ }^{134,135}$ As poly(GP) levels did not correlate with age at onset, disease duration, severity or survival, future clinical trials in patients with $C 9$ orf72 repeat expansions may benefit from the combination of poly(GP) levels as pharmacodynamic marker and NfL as a prognostic marker.

\section{$\underline{\text { Potential future fluid biomarkers }}$}

As FTLD with phosphorylated TDP-43 (pTDP-43) aggregates constitutes one of the major pathological subgroups of FTLD, pTDP-43 protein levels in CSF or blood would be an interesting biomarker, but to date, results have been contradictory. Strongly elevated CSF pTDP-43 levels have been found in a small series of $C 9$ orf72 and GRN patients, but did not differ between FTD with TDP-43 and tau pathology in a pathology-proven cohort. ${ }^{112,136}$ Quantification in CSF is challenging due to low concentrations, various isoforms and antibodies, ${ }^{112,137}$ and the development of better TDP-43 assays is warranted.

Neuroinflammation plays an important role in FTD and other neurodegenerative diseases, as it represents both a consequence of and a trigger for pathology. ${ }^{138}$ Microglia are the major immune component of the central nervous system, and are activated by damaged neurons and misfolded proteins resulting in the 
initiation of a chronic inflammatory response. ${ }^{138}$ In a study on sporadic FTD (and AD) patients, reduced levels of soluble triggering receptor expressed on myeloid cells 2 (sTREM2, a protein involved in inflammation and phagocytosis and mainly expressed by microglia) were found. ${ }^{139}$ CSF levels of YKL-40 (chitinase-3 like-1, cartilage glycoprotein-39), an inflammatory protein produced by astrocytes, have been found to be elevated in pathologically proven FTD, but also in AD, vascular dementia, normal aging and other neurological disorders such as multiple sclerosis. ${ }^{140-142}$ This is also true for glial fibrillary acidic protein, an astrocytic cytoskeletal protein, which was found to be increased in both FTD and other dementia types. ${ }^{143}$ Recently, a strong link between GRN mutations and microglial activation has been established, with excessive complement production leading to synaptic pruning. ${ }^{144}$ Promising data suggest that proteins involved in complement activation are potential biomarkers to track disease progression in GRN mutation carriers. ${ }^{144}$

Various changes in cytokines (primarily pro-inflammatory, e.g. MCP-1, IL-6, TNF- $\alpha$ ) have been found in FTD, but these also reflect aspecific mechanisms, as they are also present in AD. ${ }^{145-150}$ The role of several neuropeptides has been extensively reviewed elsewhere, ${ }^{151}$ for example neurogranin, a postsynaptic protein involved in synaptic plasticity, which was lower in FTD patients than in controls and AD patients. ${ }^{141}$ Larger, pathologically proven and genetically determined cohorts are needed for validation of these cytokines and neuropeptides.

Novel approaches are focusing on enriched protein fractions and microRNAs (miRNAs) in exosomes. Exosomes are vesicles secreted from cells; they facilitate intercellular communication and are enriched sources of biomolecules. The value of examining exosomes is supported by a small study reporting reduced synaptic protein levels in blood-derived exosomes in FTD. ${ }^{152}$ miRNAs regulate gene expression, and seem to play a role in TDP-43 and FUS pathology, but have not yet been studied as biomarkers in FTD. ${ }^{153}$

\section{$\underline{\text { Summary of fluid biomarkers }}$}


Several fluid biomarkers provide currently usable (e.g. core AD biomarkers) or promising biomarkers in FTD (e.g. NfL) (Table 1). It is likely that combinations of CSF metabolites will yield more information than single markers alone, for example a biomarker panel achieved a high sensitivity to differentiate TDP43- from tau-pathology. ${ }^{145}$ In general, more validation and longitudinal data is needed to determine the full potential of these and other candidates. Lastly, it is important to harmonize collection and analysis of fluid biomarkers, as their levels can be influenced by multiple pre-analytical and analytical factors, including sampling and storage methods, and choice and implementation of assays. ${ }^{154}$ Multicenter standardization of these procedures and quality control programs will facilitate collaborative research and the implementation of new fluid biomarkers in clinical practice.

\section{Conclusions}

Neuroimaging and fluid biomarkers are becoming increasingly important in the context of future therapeutic interventions in both sporadic and genetic forms of FTD. Several imaging and CSF biomarkers are already established (e.g. grey matter atrophy, FDG-PET and CSF AD biomarkers) and being used in clinical practice, often in the differential diagnosis of FTD. The field is moving forward in identifying gene-specific markers and finding new biomarkers for staging, predicting underlying pathology and monitoring treatment responses. For example, DTI has shown a good performance in discriminating FTD from AD, as well as demonstrating early pathological changes; NfL can differentiate FTD from controls and is a promising staging and prognostic biomarker for FTD; and genetic specific biomarkers (PGRN and DPRs) may be valuable for assessing target engagement in therapeutic trials. Importantly, combinations of biomarkers will add value in order to accurately define the FTD subtype, disease onset, as well as to monitor progression and eventually treatment response. For example, in a PGRN-enhancing trial, target engagement can be assessed by PGRN levels, but additional surrogate endpoints are needed to assess the physiological effect (i.e. reduction of neurodegeneration). 
Most alterations of these novel biomarkers have currently been demonstrated on group level and need to be validated for individual subjects, which is challenging due to the relative rarity of FTD. Multicenter research can help to increase statistical power and prove clinical utility; prime examples of longitudinal observational cohorts include GENFI (Genetic Frontotemporal Dementia Initiative), ARTFL (Advancing Research and Treatment for Frontotemporal Lobar Degeneration Consortium), LEFFTDS (Longitudinal Evaluation of Familial Frontotemporal Dementia Subjects), and a collaboration including these consortia in the FPI (FTD Prevention Initiative). Research in genetic FTD provides a unique window to study the earliest disease effects and therefore offers a high chance to identify valuable biomarkers. Despite similarities between genetic and sporadic FTD, the use of biomarkers identified in genetic cases requires validation in sporadic cohorts as biomarker profiles and trajectories may differ, alike in AD. ${ }^{155}$

Interestingly, it is increasingly emphasized that FTD, classically considered as an early-onset dementia, frequently manifests after the age of 65 years and may include clinical features suggestive of AD. ${ }^{156,157}$ This stresses the need of diagnostic biomarkers specific for FTD, as the co-occurrence of Alzheimer pathology increases with age. The value of FTD biomarkers in different age groups with comorbidities remains to be elucidated. Additionally, future research should focus on combining biomarkers (both fluid and imaging) to make optimal use of these modalities, as well as on harmonization of collection and analysis protocols to facilitate dissemination in research and clinical practices.

\section{Key points}

- Most of the validated biomarkers in frontotemporal dementia (FTD) (e.g. grey matter atrophy, FDG-PET and CSF amyloid beta ${ }_{1-42}$, phospho-tau $_{181}$, and total-tau) are used to differentiate FTD from Alzheimer's disease or controls

- New imaging biomarkers, like arterial spin labeling and diffusion tensor imaging, appear to be more sensitive to show subtle changes that precede grey matter atrophy in FTD, providing a potential role in diagnosis and monitoring 
- Promising upcoming fluid biomarkers are neurofilament light chain for staging, monitoring, and prognosis in all FTD subtypes, and dipeptide and progranulin proteins for target engagement in gene-specific forms

- There is still a need of reliable biomarkers to differentiate between tau- and TDP-pathology in light of trials on disease-modifying treatments

- Future research should focus on the multimodal combination of fluid and imaging biomarkers, as well as on the harmonizing of collection and analysis protocols

\section{Display items:}

\section{Text Boxes:}

Text Box 1. Main clinical characteristics of FTD

- BvFTD (behavioral variant FTD):

- Personality and behavioral changes (including disinhibition, apathy, loss of sympathy, perseverative behavior, abnormal appetite), and executive dysfunction

- Primary progressive aphasia (PPA): progressive prominent language difficulties that impair daily living. Subtypes:

- Semantic variant PPA (svPPA): fluent speech characterized by anomia and impaired single word comprehension

- Non-fluent variant PPA (nfvPPA): non-fluent speech with agrammatism and/or apraxia of speech

- Logopenic variant (IvPPA): non-fluent speech with word-finding difficulties in spontaneous speech and in repetition

Text Box 2. Biomarkers - requirements and applications

Requirements: (adjusted from ${ }^{6}$ )

- Able to detect fundamental feature of FTD pathology

- Validated in neuropathological confirmed FTD

- Precise

- Reliable

- Non-invasive

- Simple to perform

- Inexpensive

Applications:

- Prediction 
- Diagnostic

- Staging

- Monitoring:

- Disease progression

- Treatment response (surrogate endpoint, target engagement)

- Prognostic

Figure legends:

Figure 1. Clinical, pathological and genetic spectrum of FTD. Genetic forms have predictable pathology: GRN and C9orf72 mutations show TDP pathology whereas MAPT mutations show tau pathology. In contrast, across the clinical spectrum of FTD variable underlying pathologies and genetic forms can be found. ALS and FTD-MND phenotypes is infrequently caused by FTLD-FUS pathology or FUS mutations, for simplicity this is not included in the figure. Adapted with permission from Seelaar et al. ${ }^{158}$ ALS: amyotrophic lateral sclerosis, bvFTD: behavioral variant FTD; C9orf72: C9orf72 repeat expansions; CBD: corticobasal degeneration; FTD: frontotemporal dementia; FTLD: frontotemporal lobar degeneration; FUS: fused in sarcoma; GRN: progranulin gene mutations; MAPT: microtubule-associated protein tau mutations; MND: motor neuron disease; nfvPPA: non-fluent variant PPA; PPA: primary progressive aphasia; PSP: progressive supranuclear palsy; svPPA: semantic variant PPA; TARDP: transactive response DNA-binding protein gene; TBK-1: TANK-binding kinase 1 gene; TDP-43: transactive response DNA-binding protein 43; $V C P$ : valosin containing protein

Figure 2. Characteristic patterns of grey matter atrophy (highlighted) in different clinical and genetic subtypes of FTD. bvFTD patients show prominent frontal, insular and anterior cingulate atrophy; typical temporal atrophy in svPPA is asymmetrical (most often left); nfvPPA patients show left frontal and insular atrophy; in patients with underlying FUS-pathology, nucleus caudatus atrophy is pronounced; $G R N$ patients often show asymmetrical fronto-temporo-parietal atrophy; C9orf72 present mostly with a generalized symmetrical atrophy; and MAPT patients show marked symmetrical temporal atrophy. 
bvFTD: behavioral variant FTD; C9orf72: C9orf72 repeat expansions; FTD: frontotemporal dementia; FUS: fused in sarcoma underlying pathology; GRN: progranulin mutations; MAPT: microtubuleassociated protein tau mutations; nfvPPA: non-fluent variant PPA; PPA: primary progressive aphasia; svPPA: semantic variant PPA;

Figure 3. Imaging abnormalities in the presymptomatic stage of genetic FTD. (a) Grey matter changes adopted from the GENFI study: standardized difference between all (presymptomatic + symptomatic) mutation carriers and non-carriers in cortical grey matter volumetric imaging measures (Y-axis) versus estimated years from expected symptoms onset (X-axis); (b) DTI changes: decreased fractional anisotropy in presymptomatic GRN and MAPT mutation carriers versus controls in the uncinate fasciculus; and (c) ASL changes: lower cerebral blood flow in presymptomatic $G R N$ mutation carriers than in controls cross-sectionally at follow-up. Reproduced with permission from (a) Rohrer et al., ${ }^{30}$ (b) Dopper et al., ${ }^{37}$ and (c) Dopper et al. ${ }^{62}$

Figure 4. CSF neurofilament light chain levels. (a) CSF NfL levels in clinical FTD subtypes and other neurodegenerative disease; horizontal lines represent medians and filled squares represent patients with concomitant MND; (b) CSF NfL levels in presymptomatic and symptomatic genetic FTD of the three major genes (GRN, C9orf72 and MAPT), including two subjects who converted from the presymptomatic to symptomatic stage (connecting line), filled squares represent patients with concomitant MND; and (c) Kaplan-Meier survival curves stratified for CSF NfL levels in tertiles; upper light-grey line represents low CSF NfL levels, middle dark-grey line represents intermediate CSF NfL levels, and lower black line represents high CSF NfL levels. Adapted with permission from (a) Scherling et al., ${ }^{114}$ and (b+c) Meeter et al. ${ }^{115}$ Permission obtained from Scherling and Meeter; permission necessary from (a) Annals of Neurology, and $(b+c)$ Annals of Clinical and Translational Neurology.

AD: Alzheimer's disease; bvFTD: behavioral variant FTD; C9orf72: C9orf72 repeat expansions; CBS: corticobasal syndrome; CSF: cerebrospinal fluid; GRN: progranulin mutations; MAPT: microtubule- 
associated protein tau mutations; MND: motor neuron disease; NC: normal controls; NC2: healthy at risk for a genetic mutation subjects; NfL: neurofilament light chain; nfvPPA: non fluent variant PPA; PD: Parkinson's disease; PPA: primary progressive aphasia; PSP: progressive supranuclear palsy; svPPA: semantic variant PPA.

Figure 5. Genetic specific fluid biomarkers. (a) plasma PGRN levels are significantly lower, without overlap, in carriers of GRN mutations (presymptomatic carriers, ASX GRN+, and symptomatic carriers, $\mathrm{SX}$ GRN+) than in controls (GRN-), [(b+c) confidential panels, will be published in March] (b) CSF poly(GP) levels are significantly higher in $C 9$ orf 72 repeat expansion carriers (C9+) than non-carriers (C91), (c) already in the presymptomatic stage (ASX) when compared to the symptomatic stage (SX). Adapted with permission from (a) Meeter et al., ${ }^{129}$ and (b+c) adopted from Gendron et al. ${ }^{135}$ Horizontal red lines represent the sample medians in a given group. This figure is optional; in our opinion it is very illustrative, however it exceeds the maximum of 7 display items. Lay-out, including terminology, can be adjusted once the poly(GP) figures are published and permission to adapt is obtained.

ASX: asymptomatic (presymptomatic) carrier; C9+: C9orf72 repeat expansion carrier; C9-: non-carriers of a C9orf72 repeat expansion; CSF: cerebrospinal fluid; GRN+: progranulin gene mutation carrier; $G R N$-: non-GRN-carriers; PGRN: progranulin protein; poly(GP): glycine-proline repeating protein; SX: symptomatic carrier

\section{$\underline{\text { Tables }}$}

\begin{tabular}{|c|c|c|c|c|c|c|}
\hline \multirow[t]{3}{*}{ Biomarker } & \multicolumn{6}{|c|}{ Application } \\
\hline & \multicolumn{2}{|c|}{ Diagnosis } & \multicolumn{2}{|c|}{$\begin{array}{l}\text { Staging and monitoring } \\
\text { disease progression }\end{array}$} & \multirow{2}{*}{ Prognosis } & \multirow{2}{*}{$\begin{array}{c}\text { Monitoring } \\
\text { treatment } \\
\text { response }\end{array}$} \\
\hline & $F T D$ vs $A D$ & $\begin{array}{c}\text { FTD subtypes } \\
\text { (clinical/genetic } \\
\text { /pathologic) }\end{array}$ & Symptomatic & $\begin{array}{c}\text { Presymptom } \\
\text { atic }\end{array}$ & & \\
\hline
\end{tabular}




\begin{tabular}{|c|c|c|c|c|c|c|}
\hline $\begin{array}{l}\text { Grey matter atrophy } \\
\text { DTI } \\
\text { FDG-PET } \\
\text { Tau-PET } \\
\text { ASL } \\
\text { RS-fMRI }\end{array}$ & $\begin{array}{c}++ \\
++ \\
++ \\
+ \\
++ \\
+\end{array}$ & $\begin{array}{c}++ \\
+ \\
++ \\
+ \\
+ \\
+\end{array}$ & $\begin{array}{l}+ \\
+ \\
+ \\
+ \\
+\end{array}$ & $\begin{array}{l}+ \\
+ \\
+ \\
+ \\
+\end{array}$ & & $\begin{array}{l}+ \\
+ \\
+\end{array}$ \\
\hline \multicolumn{7}{|l|}{ Fluid biomarkers } \\
\hline $\begin{array}{l}\text { p-tau, t-tau and } A \beta_{1-42} \\
\text { NfL } \\
\text { PGRN } \\
\text { Poly(GP) }\end{array}$ & $\begin{array}{c}++ \\
+\end{array}$ & $\begin{array}{c}+ \\
+ \\
++ \\
+\end{array}$ & ++ & + & $\begin{array}{l}+^{\mathrm{a}} \\
++\end{array}$ & $\begin{array}{l}+ \\
+ \\
+\end{array}$ \\
\hline \multicolumn{7}{|c|}{ 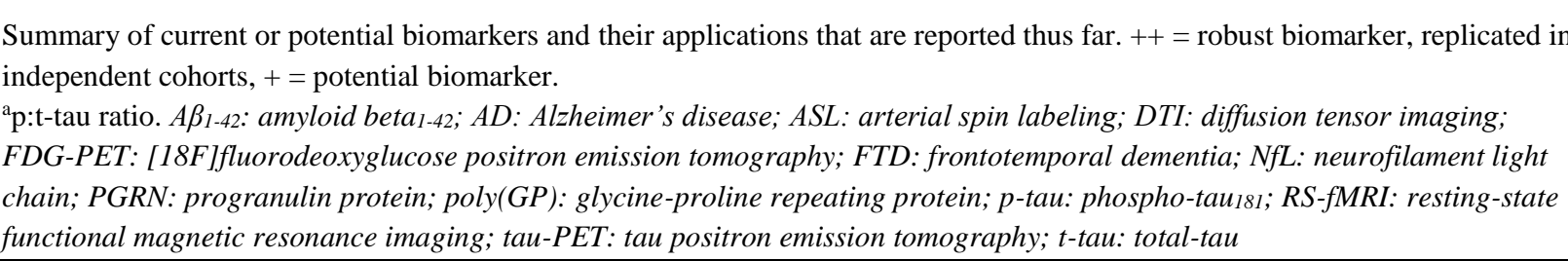 } \\
\hline
\end{tabular}

\section{Acknowledgments}

We would like to thank Serge A. Rombouts, Meike W. Vernooij, and Rebecca M. Steketee for their constructive comments on subsections of this review. We thank Carole Scherling and Adam L. Boxer for the raw NfL data to assembly Figure 4, and Tania F. Gendron and Leonard Petrucelli for the consent to use the poly(GP) figures.

LHM, LDK and JCvS received funding from a Memorable grant from Deltaplan Dementie (The Netherlands Organisation for Health Research and Development, and the Netherlands Alzheimer Foundation, grant nr. 70-73305-98-105), and the European Joint Programme - Neurodegenerative Disease Research (JPND, PreFrontALS). LHM is supported by Alzheimer Nederland (grant nr. WE.09-2014-04). $\mathrm{JCvS}$ is supported by the Dioraphte Foundation. LDK is supported by The Bluefield Project. JDR is supported by an MRC Clinician Scientist Fellowship (MR/M008525/1) and has received funding from the NIHR Rare Disease Translational Research Collaboration.

\section{Authors contributions}

LHM, LDK, JDR and JCvS performed the literature review and drafted and revised the manuscript and figures. 


\section{Competing interests}

The authors declare no competing financial interests.

\section{Author biographies}

Lieke H. Meeter is a PhD student at the Alzheimer Center (Erasmus Medical Center) in Rotterdam, the Netherlands. She obtained her medical degree at the University of Utrecht in 2011 and has worked as a medical doctor since, with interest in neurology and cognition. In 2014 she started her $\mathrm{PhD}$ research, which focusses on fluid biomarkers in genetic and sporadic FTD. Most of her projects are in international collaborations such as the Genetic FTD Initiative (GENFI).

Laura Donker Kaat is a neurologist and resident in clinical genetics; she has a special interest in the genetics of adult onset neurological diseases. During her $\mathrm{PhD}$, she worked on the clinical and genetic aspects of Progressive Supranuclear Palsy. In addition to her work as a resident in clinical genetics, she is involved in scientific projects on hereditary FTD at the Alzheimer Center in Rotterdam.

Jonathan D. Rohrer: Dr Rohrer's research focuses on the neuroimaging and neuropsychology of FTD, particularly in relation to their underlying genetic causes. Research in the field of FTD has led to the publication of over 120 Pubmed-referenced papers and he has spoken at a number of international conferences about the work. Since 2011 he has co-ordinated the Genetic FTD Initiative, GENFI, a multicentre cohort study of presymptomatic genetic FTD, and has been the clinical lead for the International FTD GWAS Consortium. He has also set up FTD UK, an annual scientific meeting of UK researchers who work in the FTD field (running since 2011), and runs a website dedicated to providing research updates to the general public about FTD: FTD talk.

John C. van Swieten: Dr. John van Swieten's research focus has been on FTD since his first paper on hereditary FTD linked to chromosome 17q21-22 in 1996. He was involved in the identification of MAPT mutations, and his work includes clinical, pathological and genetic aspects of dementia and related 
disorders (> 200 papers). He currently coordinates research on neuroimaging and fluid biomarkers in a large Dutch cohort of presymptomatic carriers, and takes part in the European GENFI initiative. Dr. John van Swieten has identified for the first time mutations in two genes (PRKARIB, FGF14) in families with hereditary dementia-parkinsonism and with cerebellar degeneration.

\section{References}

1. Rascovsky, K. et al. Sensitivity of revised diagnostic criteria for the behavioural variant of frontotemporal dementia. Brain 134, 2456-77 (2011).

2. Gorno-Tempini, M. L. et al. Classification of primary progressive aphasia and its variants. Neurology 76, 1006-14 (2011).

3. Lashley, T., Rohrer, J. D., Mead, S. \& Revesz, T. Review: An update on clinical, genetic and pathological aspects of frontotemporal lobar degenerations. Neuropathol. Appl. Neurobiol. 41, 858-881 (2015).

4. Josephs, K. A. et al. Neuropathological background of phenotypical variability in frontotemporal dementia. Acta Neuropathol. 122, 137-153 (2011).

5. Pottier, C., Ravenscroft, T. A., Sanchez-Contreras, M. \& Rademakers, R. Genetics of FTLD: overview and what else we can expect from genetic studies. J. Neurochem. 138, 32-53 (2016).

6. Consensus report of the Working Group on: "Molecular and Biochemical Markers of Alzheimer's Disease". The Ronald and Nancy Reagan Research Institute of the Alzheimer's Association and the National Institute on Aging Working Group. Neurobiol. Aging 19, 109-16 (1998).

7. Gordon, E., Rohrer, J. D. \& Fox, N. C. Advances in neuroimaging in frontotemporal dementia. J. Neurochem. 138, 193-210 (2016).

8. Rohrer, J. D. \& Rosen, H. J. Neuroimaging in frontotemporal dementia. Int. Rev. Psychiatry 25, 221-229 (2013).

9. Whitwell, J. L. \& Josephs, K. A. Neuroimaging in frontotemporal lobar degenerationpredicting molecular pathology. Nat. Rev. Neurol. 8, 131-142 (2012).

10. Diehl-Schmid, J., Onur, O. A., Kuhn, J., Gruppe, T. \& Drzezga, A. Imaging frontotemporal lobar degeneration. Curr. Neurol. Neurosci. Rep. 14, 489 (2014).

11. Harper, L. et al. MRI visual rating scales in the diagnosis of dementia: Evaluation in 184 post-mortem confirmed cases. Brain 139, 1211-1225 (2016).

12. Schroeter, M. L. et al. Conceptualizing neuropsychiatric diseases with multimodal datadriven meta-analyses - The case of behavioral variant frontotemporal dementia. Cortex $\mathbf{5 7}$, 
22-37 (2014).

13. Pan, P. L. et al. Gray matter atrophy in behavioral variant frontotemporal dementia: a meta-analysis of voxel-based morphometry studies. Dement. Geriatr. Cogn. Disord. 33, 141-8 (2012).

14. Seeley, W. W. et al. Frontal paralimbic network atrophy in very mild behavioral variant frontotemporal dementia. Arch. Neurol. 65, 249-55 (2008).

15. Whitwell, J. L. et al. Distinct anatomical subtypes of the behavioural variant of frontotemporal dementia: A cluster analysis study. Brain 132, 2932-2946 (2009).

16. Whitwell, J. L. et al. Neuroimaging signatures of frontotemporal dementia genetics: C9ORF72, tau, progranulin and sporadics. Brain 135, 794-806 (2012).

17. Schroeter, M. L., Raczka, K., Neumann, J. \& Yves von Cramon, D. Towards a nosology for frontotemporal lobar degenerations-A meta-analysis involving 267 subjects. Neuroimage 36, 497-510 (2007).

18. Gorno-Tempini, M. et al. Cognition and anatomy in three variants of primary progressive aphasia. Ann. Neurol. 55, 335-346 (2004).

19. Rogalski, E. et al. Progression of language decline and cortical atrophy in subtypes of primary progressive aphasia. Neurology 76, 1804-10 (2011).

20. Kumfor, F. et al. On the right side? A longitudinal study of left- versus right-lateralized semantic dementia. Brain 139, 986-98 (2016).

21. Rohrer, J. D. et al. Rates of hemispheric and lobar atrophy in the language variants of frontotemporal lobar degeneration. J. Alzheimer's Dis. 30, 407-411 (2012).

22. Rohrer, J. D. et al. Patterns of cortical thinning in the language variants of frontotemporal lobar degeneration. Neurology 72, 1562-1569 (2009).

23. Rohrer, J. D. et al. Distinct profiles of brain atrophy in frontotemporal lobar degeneration caused by progranulin and tau mutations. Neuroimage 53, 1070-1076 (2010).

24. Deters, K. D. et al. Cerebral hypometabolism and grey matter density in MAPT intron 10 +3 mutation carriers. Am. J. Neurodegener. Dis. 3, 103-14 (2014).

25. Sha, S. J. et al. Frontotemporal dementia due to C9ORF72 mutations: clinical and imaging features. Neurology 79, 1002-11 (2012).

26. Lee, S. E. et al. Altered network connectivity in frontotemporal dementia with C9orf72 hexanucleotide repeat expansion. Brain 137, 3047-3060 (2014).

27. Seelaar, H. et al. Frequency of ubiquitin and FUS-positive, TDP-43-negative frontotemporal lobar degeneration. J. Neurol. 257, 747-53 (2010).

28. Josephs, K. A. et al. Caudate atrophy on MRI is a characteristic feature of FTLD-FUS. Eur. J. Neurol. 17, 969-975 (2010). 
29. Whitwell, J. L. et al. Brain atrophy over time in genetic and sporadic frontotemporal dementia: a study of 198 serial magnetic resonance images. Eur. J. Neurol. 22, 745-52 (2015).

30. Rohrer, J. D. et al. Presymptomatic cognitive and neuroanatomical changes in genetic frontotemporal dementia in the Genetic Frontotemporal dementia Initiative (GENFI) study: a cross-sectional analysis. Lancet. Neurol. 14, 253-62 (2015).

31. Caroppo, P. et al. Lateral Temporal Lobe: An Early Imaging Marker of the Presymptomatic GRN Disease? J. Alzheimer's Dis. 47, 751-759 (2015).

32. Lu, P. H. et al. Regional differences in white matter breakdown between frontotemporal dementia and early-onset Alzheimer's disease. J. Alzheimer's Dis. 39, 261-269 (2014).

33. Agosta, F. et al. MRI signatures of the frontotemporal lobar degeneration continuum. Hum. Brain Mapp. 36, 2602-2614 (2015).

34. Mahoney, C. J. et al. Profiles of white matter tract pathology in frontotemporal dementia. Hum. Brain Mapp. 35, 4163-4179 (2014).

35. Lam, B. Y. K., Halliday, G. M., Irish, M., Hodges, J. R. \& Piguet, O. Longitudinal white matter changes in frontotemporal dementia subtypes. Hum. Brain Mapp. 35, 3547-3557 (2014).

36. Mahoney, C. J. et al. White matter tract signatures of the progressive aphasias. Neurobiol. Aging 34, 1687-1699 (2013).

37. Dopper, E. G. P. et al. Structural and functional brain connectivity in presymptomatic familial frontotemporal dementia. Neurology 83, e19-26 (2014).

38. Schwindt, G. C. et al. Whole-brain white matter disruption in semantic and nonfluent variants of primary progressive aphasia. Hum. Brain Mapp. 34, 973-984 (2013).

39. Zhang, Y. et al. White matter damage in frontotemporal dementia and Alzheimer's disease measured by diffusion MRI. Brain 132, 2579-92 (2009).

40. McMillan, C. T. et al. White matter imaging contributes to the multimodal diagnosis of frontotemporal lobar degeneration. Neurology 78, 1761-1768 (2012).

41. Mahoney, C. J. et al. Longitudinal diffusion tensor imaging in frontotemporal dementia. Ann. Neurol. 77, 33-46 (2015).

42. Zhang, Y. et al. MRI signatures of brain macrostructural atrophy and microstructural degradation in frontotemporal lobar degeneration subtypes. J. Alzheimer's Dis. 33, 431444 (2013).

43. Tu, S., Leyton, C. E., Hodges, J. R., Piguet, O. \& Hornberger, M. Divergent longitudinal propagation of white matter degradation in logopenic and semantic variants of primary progressive aphasia. J. Alzheimer's Dis. 49, 853-861 (2016).

44. Agosta, F. et al. White matter damage in frontotemporal lobar degeneration spectrum. 
Cereb. Cortex 22, 2705-2714 (2012).

45. Mcmillan, C. T. et al. The power of neuroimaging biomarkers for screening frontotemporal dementia. Hum. Brain Mapp. 35, 4827-4840 (2014).

46. McMillan, C. T. et al. White matter imaging helps dissociate tau from TDP-43 in frontotemporal lobar degeneration. J. Neurol. Neurosurg. Psychiatry 84, 949-55 (2013).

47. Borroni, B. et al. Brain magnetic resonance imaging structural changes in a pedigree of asymptomatic progranulin mutation carriers. Rejuvenation Res. 11, 585-595 (2008).

48. Brewer, J. B. Fully-automated volumetric MRI with normative ranges: Translation to clinical practice. Behav. Neurol. 21, 21-28 (2009).

49. Jack, C. R. et al. Hypothetical model of dynamic biomarkers of the Alzheimer's pathological cascade. Lancet Neurol. 9, 119-128 (2010).

50. Morbelli, S. et al. Mapping brain morphological and functional conversion patterns in predementia late-onset bvFTD. Eur. J. Nucl. Med. Mol. Imaging 43, 1337-1347 (2016).

51. Verfaillie, S. C. J. et al. Cerebral perfusion and glucose metabolism in Alzheimer's disease and frontotemporal dementia: two sides of the same coin? Eur. Radiol. 25, 30503059 (2015).

52. Dukart, J. et al. Combined evaluation of FDG-PET and MRI improves detection and differentiation of dementia. PLoS One 6, e18111 (2011).

53. Tosun, D. et al. Diagnostic utility of ASL-MRI and FDG-PET in the behavioral variant of FTD and AD. Ann. Clin. Transl. Neurol. 3, 740-751 (2016).

54. Vijverberg, E. G. B. et al. Diagnostic Accuracy of MRI and Additional [18F]FDG-PET for Behavioral Variant Frontotemporal Dementia in Patients with Late Onset Behavioral Changes. J. Alzheimer's Dis. 53, 1287-1297 (2016).

55. Buhour, M.-S. et al. Pathophysiology of the behavioral variant of frontotemporal lobar degeneration: A study combining MRI and FDG-PET. Brain Imaging Behav. 1-13 (2016). doi:10.1007/s11682-016-9521-x

56. Diehl-Schmid, J. et al. Decline of cerebral glucose metabolism in frontotemporal dementia: a longitudinal 18F-FDG-PET-study. Neurobiol. Aging 28, 42-50 (2007).

57. Cerami, C. et al. The Role of Single-Subject Brain Metabolic Patterns in the Early Differential Diagnosis of Primary Progressive Aphasias and in Prediction of Progression to Dementia. J. Alzheimers. Dis. 55, 183-197 (2017).

58. Diehl-Schmid, J. et al. Longitudinal changes of cerebral glucose metabolism in semantic dementia. Dement. Geriatr. Cogn. Disord. 22, 346-351 (2006).

59. Jacova, C. et al. Anterior brain glucose hypometabolism predates dementia in progranulin mutation carriers. Neurology 81, 1322-1331 (2013). 
60. Cistaro, A. et al. The metabolic signature of C9ORF72-related ALS: FDG PET comparison with nonmutated patients. Eur. J. Nucl. Med. Mol. Imaging 41, 844-852 (2014).

61. Alsop, D. C. et al. Recommended implementation of arterial spin-labeled Perfusion mri for clinical applications: A consensus of the ISMRM Perfusion Study group and the European consortium for ASL in dementia. Magn. Reson. Med. 73, 102-116 (2015).

62. Dopper, E. G. P. P. et al. Cerebral blood flow in presymptomatic MAPT and GRN mutation carriers: A longitudinal arterial spin labeling study. NeuroImage. Clin. 12, 460-5 (2016).

63. Steketee, R. M. E. et al. Early-stage differentiation between presenile Alzheimer's disease and frontotemporal dementia using arterial spin labeling MRI. Eur. Radiol. 26, 244-53 (2016).

64. Binnewijzend, M. A. A. et al. Distinct perfusion patterns in Alzheimer's disease, frontotemporal dementia and dementia with Lewy bodies. Eur. Radiol. 24, 2326-2333 (2014).

65. Du, A. T. et al. Hypoperfusion in frontotemporal dementia and Alzheimer disease by arterial spin labeling MRI. Neurology 67, 1215-20 (2006).

66. Premi, E. et al. Multimodal FMRI resting-state functional connectivity in granulin mutations: the case of fronto-parietal dementia. PLoS One 9, e106500 (2014).

67. Whitwell, J. L. et al. Altered functional connectivity in asymptomatic MAPT subjects: a comparison to bvFTD. Neurology 77, 866-74 (2011).

68. Zhou, J. et al. Divergent network connectivity changes in behavioural variant frontotemporal dementia and Alzheimer's disease. Brain 133, 1352-1367 (2010).

69. Filippi, M. et al. Functional network connectivity in the behavioral variant of frontotemporal dementia. Cortex. 49, 2389-401 (2013).

70. Day, G. S. et al. Salience Network Resting-State Activity. JAMA Neurol. 70, 1249-1253 (2013).

71. Seeley, W. W. et al. Divergent social functioning in behavioral variant frontotemporal dementia and Alzheimer disease: reciprocal networks and neuronal evolution. Alzheimer Dis Assoc Disord 21, S50-7 (2007).

72. Rytty, R. et al. GroupICA dual regression analysis of resting state networks in a behavioral variant of frontotemporal dementia. Front. Hum. Neurosci. 7, 461 (2013).

73. Hafkemeijer, A. et al. Resting state functional connectivity differences between behavioral variant frontotemporal dementia and Alzheimer's disease. Front. Hum. Neurosci. 9, 474 (2015).

74. Farb, N. A. S. et al. Abnormal network connectivity in frontotemporal dementia: Evidence 
for prefrontal isolation. Cortex 49, 1856-1873 (2013).

75. Pievani, M., de Haan, W., Wu, T., Seeley, W. W. \& Frisoni, G. B. Functional network disruption in the degenerative dementias. Lancet Neurol. 10, 829-843 (2011).

76. Guo, C. C. et al. Anterior temporal lobe degeneration produces widespread networkdriven dysfunction. Brain 136, 2979-2991 (2013).

77. Agosta, F. et al. Disrupted brain connectome in semantic variant of primary progressive aphasia. Neurobiol. Aging 35, 2646-2655 (2014).

78. Borroni, B. et al. Granulin mutation drives brain damage and reorganization from preclinical to symptomatic FTLD. Neurobiol. Aging 33, 2506-2520 (2012).

79. Premi, E. et al. Looking for Neuroimaging Markers in Frontotemporal Lobar Degeneration Clinical Trials: A Multi-Voxel Pattern Analysis Study in Granulin Disease. J. Alzheimers. Dis. 51, 249-62 (2016).

80. Ishii, K. PET approaches for diagnosis of dementia. Am. J. Neuroradiol. 35, 2030-8 (2014).

81. Laforce, R. et al. Parallel ICA of FDG-PET and PiB-PET in three conditions with underlying Alzheimer's pathology. NeuroImage Clin. 4, 508-516 (2014).

82. Rabinovici, G. D. et al. Abeta amyloid and glucose metabolism in three variants of primary progressive aphasia. Ann. Neurol. 64, 388-401 (2008).

83. Matías-Guiu, J. A. et al. Amyloid and FDG-PET study of logopenic primary progressive aphasia: evidence for the existence of two subtypes. J. Neurol. 262, 1463-1472 (2015).

84. Whitwell, J. L. et al. Clinical and neuroimaging biomarkers of amyloid-negative logopenic primary progressive aphasia. Brain Lang. 142, 45-53 (2015).

85. Leyton, C. E., Ballard, K. J., Piguet, O. \& Hodges, J. R. Phonologic errors as a clinical marker of the logopenic variant of PPA. Neurology 82, 1620-7 (2014).

86. Villemagne, V. L., Fodero-Tavoletti, M. T., Masters, C. L. \& Rowe, C. C. Tau imaging: Early progress and future directions. Lancet Neurol. 14, 114-124 (2015).

87. Smith, R. et al. 18F-AV-1451 tau PET imaging correlates strongly with tau neuropathology in MAPT mutation carriers. Brain 139, 2372-9 (2016).

88. Smith, R. et al. Tau neuropathology correlates with FDG-PET, but not AV-1451-PET, in progressive supranuclear palsy. Acta Neuropathol. 133, 149-151 (2017).

89. Marquié, M. et al. Pathologic correlations of [F-18]-AV-1451 imaging in non-Alzheimer tauopathies. Ann. Neurol. 1, 1-29 (2016).

90. Ono, M. et al. Distinct binding of PET ligands PBB3 and AV-1451 to tau fibril strains in neurodegenerative tauopathies. Brain 1, aww339 (2017). 
91. Ishiki, A. et al. Tau imaging with [(18) F]THK-5351 in progressive supranuclear palsy. Eur. J. Neurol. 24, 130-136 (2017).

92. Kikuchi, A. et al. In vivo visualization of tau deposits in corticobasal syndrome by 18 FTHK5351 PET. Neurology 87, 2309-2316 (2016).

93. Bron, E. E. et al. Multiparametric computer-aided differential diagnosis of Alzheimer's disease and frontotemporal dementia using structural and advanced MRI. Eur. Radiol. (2016). doi:10.1007/s00330-016-4691-x

94. Tahmasian, M. et al. Based on the Network Degeneration Hypothesis: Separating Individual Patients with Different Neurodegenerative Syndromes in a Preliminary Hybrid PET/MR Study. J. Nucl. Med. 57, 410-5 (2016).

95. Olsson, B. et al. CSF and blood biomarkers for the diagnosis of Alzheimer's disease: A systematic review and meta-analysis. Lancet Neurol. 4422, 1-12 (2016).

96. Rivero-Santana, A. et al. Cerebrospinal Fluid Biomarkers for the Differential Diagnosis between Alzheimer's Disease and Frontotemporal Lobar Degeneration: Systematic Review, HSROC Analysis, and Confounding Factors. J. Alzheimers. Dis. 55, 625-644 (2017).

97. Struyfs, H. et al. Diagnostic Accuracy of Cerebrospinal Fluid Amyloid- $\beta$ Isoforms for Early and Differential Dementia Diagnosis. J. Alzheimers. Dis. 45, 813-22 (2015).

98. Janelidze, S. et al. CSF A $\beta 42 / A \beta 40$ and A $\beta 42 / A \beta 38$ ratios: better diagnostic markers of Alzheimer disease. Ann. Clin. Transl. Neurol. 3, 154-65 (2016).

99. Santangelo, R. et al. Cerebrospinal fluid biomarkers can play a pivotal role in the diagnostic work up of primary progressive aphasia. J. Alzheimers. Dis. 43, 1429-40 (2015).

100. Hu, W. T. et al. Multimodal predictors for Alzheimer disease in nonfluent primary progressive aphasia. Neurology 75, 595-602 (2010).

101. Teichmann, M. et al. Deciphering logopenic primary progressive aphasia: A clinical, imaging and biomarker investigation. Brain 136, 3474-3488 (2013).

102. Paraskevas, G. P. et al. Cerebrospinal Fluid Biomarkers as a Diagnostic Tool of the Underlying Pathology of Primary Progressive Aphasia. J. Alzheimers. Dis. 55, 1453-1461 (2017).

103. Toledo, J. B. et al. CSF biomarkers cutoffs: The importance of coincident neuropathological diseases. Acta Neuropathol. 124, 23-35 (2012).

104. Kamalainen, A. et al. Cerebrospinal fluid biomarkers for Alzheimer's disease in patients with frontotemporal lobar degeneration and amyotrophic lateral sclerosis with the C9ORF72 repeat expansion. Dement Geriatr Cogn Disord 39, 287-293 (2015).

105. Wallon, D. et al. Definite behavioral variant of frontotemporal dementia with C9ORF72 
expansions despite positive Alzheimer's disease cerebrospinal fluid biomarkers. $J$. Alzheimer's Dis. 32, 19-22 (2012).

106. Carecchio, M. et al. Cerebrospinal fluid biomarkers in progranulin mutations carriers. $J$. Alzheimer's Dis. 27, 781-790 (2011).

107. Rosso, S. M. et al. Total tau and phosphorylated tau 181 levels in the cerebrospinal fluid of patients with frontotemporal dementia due to P301L and G272V tau mutations. Arch. Neurol. 60, 1209-1213 (2003).

108. Bian, H. et al. CSF biomarkers in frontotemporal lobar degeneration with known pathology. Neurology 70, 1827-35 (2008).

109. Hu, W. T. et al. Reduced CSF p-Tau181 to Tau ratio is a biomarker for FTLD-TDP. Neurology 81, 1945-52 (2013).

110. Borroni, B. et al. Csf p-tau181/tau ratio as biomarker for TDP pathology in frontotemporal dementia. Amyotroph. Lateral Scler. Frontotemporal Degener. 16, 86-91 (2015).

111. Pijnenburg, Y. A. L., Verwey, N. A., van der Flier, W. M., Scheltens, P. \& Teunissen, C. E. Discriminative and prognostic potential of cerebrospinal fluid phosphoTau/tau ratio and neurofilaments for frontotemporal dementia subtypes. Alzheimer's Dement. Diagnosis, Assess. Dis. Monit. 1, 505-512 (2015).

112. Kuiperij, H. B. et al. Tau Rather than TDP-43 Proteins are Potential Cerebrospinal Fluid Biomarkers for Frontotemporal Lobar Degeneration Subtypes: A Pilot Study. $J$. Alzheimers. Dis. 55, 585-595 (2017).

113. Yuan, A. et al. Neurofilament subunits are integral components of synapses and modulate neurotransmission and behavior in vivo. Mol. Psychiatry 20, 986-94 (2015).

114. Scherling, C. S. et al. Cerebrospinal fluid neurofilament concentration reflects disease severity in frontotemporal degeneration. Ann. Neurol. 75, 116-126 (2014).

115. Meeter, L. H. et al. Neurofilament light chain: a biomarker for genetic frontotemporal dementia. Ann. Clin. Transl. Neurol. 3, 623-636 (2016).

116. Rohrer, J. D. et al. Serum neurofilament light chain protein is a measure of disease intensity in frontotemporal dementia. Neurology 87, 1329-36 (2016).

117. Skillback, T. et al. CSF neurofilament light differs in neurodegenerative diseases and predicts severity and survival. Neurology 83, 1945-1953 (2014).

118. Landqvist Waldö, M. et al. Cerebrospinal fluid neurofilament light chain protein levels in subtypes of frontotemporal dementia. BMC Neurol. 13, 54 (2013).

119. Wilke, C. et al. Neurofilament light chain in FTD is elevated not only in cerebrospinal fluid, but also in serum. J. Neurol. Neurosurg. Psychiatry 87, 1270-1272 (2016).

120. Lu, C.-H. et al. Neurofilament light chain: A prognostic biomarker in amyotrophic lateral sclerosis. Neurology 84, 2247-57 (2015). 
121. Rojas, J. C. et al. Plasma neurofilament light chain predicts progression in progressive supranuclear palsy. Ann. Clin. Transl. Neurol. 3, 216-25 (2016).

122. Hansson, O. et al. Blood-based NfL: A biomarker for differential diagnosis of parkinsonian disorder. Neurology 88, 930-937 (2017).

123. Steinacker, P. et al. Diagnostic and prognostic significance of neurofilament light chain NF-L, but not progranulin and S100B, in the course of amyotrophic lateral sclerosis: Data from the German MND-net. Amyotroph. Lateral Scler. Front. Degener. 18, 112-119 (2017).

124. Bacioglu, M. et al. Neurofilament Light Chain in Blood and CSF as Marker of Disease Progression in Mouse Models and in Neurodegenerative Diseases. Neuron 91, 56-66 (2016).

125. Van Damme, P. et al. Progranulin functions as a neurotrophic factor to regulate neurite outgrowth and enhance neuronal survival. J. Cell Biol. 181, 37-41 (2008).

126. Baker, M. et al. Mutations in progranulin cause tau-negative frontotemporal dementia linked to chromosome 17. Nature 442, 916-9 (2006).

127. Finch, N. et al. Plasma progranulin levels predict progranulin mutation status in frontotemporal dementia patients and asymptomatic family members. Brain 132, 583-91 (2009).

128. Ghidoni, R. et al. Optimal plasma progranulin cutoff value for predicting null progranulin mutations in neurodegenerative diseases: a multicenter Italian study. Neurodegener. Dis. 9, 121-7 (2012).

129. Meeter, L. H. H. et al. Progranulin Levels in Plasma and Cerebrospinal Fluid in Granulin Mutation Carriers. Dement. Geriatr. Cogn. Dis. Extra 6, 330-340 (2016).

130. Cenik, B. et al. Suberoylanilide hydroxamic acid (vorinostat) up-regulates progranulin transcription: rational therapeutic approach to frontotemporal dementia. J. Biol. Chem. 286, 16101-8 (2011).

131. Feneberg, E. et al. Progranulin as a candidate biomarker for therapeutic trial in patients with ALS and FTLD. J. Neural Transm. 123, 289-96 (2016).

132. Mori, K. et al. The C9orf72 GGGGCC repeat is translated into aggregating dipeptiderepeat proteins in FTLD/ALS. Science 339, 1335-8 (2013).

133. Jiang, J. et al. Gain of Toxicity from ALS/FTD-Linked Repeat Expansions in C9ORF72 Is Alleviated by Antisense Oligonucleotides Targeting GGGGCC-Containing RNAs. Neuron 90, 535-550 (2016).

134. Su, Z. et al. Discovery of a Biomarker and Lead Small Molecules to Target r(GGGGCC)Associated Defects in c9FTD/ALS. Neuron 83, 1043-1050 (2014).

135. Gendron, T. F. et al. Poly(GP) proteins as a pharmacodynamic biomarker for C9ORF72- 
associated amyotrophic lateral sclerosis. Sci. Transl. Med. Accepted for publication (2017).

136. Suárez-Calvet, M. et al. Plasma phosphorylated TDP-43 levels are elevated in patients with frontotemporal dementia carrying a C9orf72 repeat expansion or a GRN mutation. $J$. Neurol. Neurosurg. Psychiatry 43, 1-8 (2013).

137. Goossens, J. et al. TDP-43 as a possible biomarker for frontotemporal lobar degeneration: a systematic review of existing antibodies. Acta Neuropathol. Commun. 3, 15 (2015).

138. Heneka, M. T., Kummer, M. P. \& Latz, E. Innate immune activation in neurodegenerative disease. Nat. Rev. Immunol. 14, 463-477 (2014).

139. Kleinberger, G. et al. TREM 2 mutations implicated in neurodegeneration impair cell surface transport and phagocytosis. Sci. Transl. Med. 6, 243 ra86 (2014).

140. Teunissen, C. E. et al. Novel diagnostic cerebrospinal fluid biomarkers for pathologic subtypes of frontotemporal dementia identified by proteomics. Alzheimer's Dement. Diagnosis, Assess. Dis. Monit. 2, 86-94 (2016).

141. Janelidze, S. et al. Cerebrospinal fluid neurogranin and YKL-40 as biomarkers of Alzheimer's disease. Ann. Clin. Transl. Neurol. 3, 12-20 (2016).

142. Alcolea, D. et al. Relationship between $\beta$-Secretase, inflammation and core cerebrospinal fluid biomarkers for Alzheimer's disease. J. Alzheimers. Dis. 42, 157-67 (2014).

143. Ishiki, A. et al. Glial fibrillar acidic protein in the cerebrospinal fluid of Alzheimer's disease, dementia with Lewy bodies, and frontotemporal lobar degeneration. $J$. Neurochem. 136, 258-261 (2016).

144. Lui, H. et al. Progranulin Deficiency Promotes Circuit-Specific Synaptic Pruning by Microglia via Complement Activation. Cell 165, 921-935 (2016).

145. Hu, W. T. et al. Novel CSF biomarkers for frontotemporal lobar degenerations. Neurology 75, 2079-86 (2010).

146. Galimberti, D. et al. Intrathecal levels of IL-6, IL-11 and LIF in Alzheimer's disease and frontotemporal lobar degeneration. J. Neurol. 255, 539-44 (2008).

147. Rentzos, M. et al. Interleukin-12 is reduced in cerebrospinal fluid of patients with Alzheimer's disease and frontotemporal dementia. J. Neurol. Sci. 249, 110-4 (2006).

148. Galimberti, D. et al. Intrathecal chemokine levels in Alzheimer disease and frontotemporal lobar degeneration. Neurology 66, 146-7 (2006).

149. Gibbons, L. et al. Plasma levels of progranulin and interleukin-6 in frontotemporal lobar degeneration. Neurobiol. Aging 36, 1603.e1-4 (2015).

150. Galimberti, D. et al. Inflammatory molecules in Frontotemporal Dementia: Cerebrospinal fluid signature of progranulin mutation carriers. Brain. Behav. Immun. 49, 182-187 (2015). 
151. Oeckl, P., Steinacker, P., Feneberg, E. \& Otto, M. Cerebrospinal fluid proteomics and protein biomarkers in frontotemporal lobar degeneration: Current status and future perspectives. Biochim. Biophys. Acta - Proteins Proteomics 1854, 757-768 (2015).

152. Goetzl, E. J. et al. Decreased synaptic proteins in neuronal exosomes of frontotemporal dementia and Alzheimer's disease. FASEB J. 30, 4141-4148 (2016).

153. Eitan, C. \& Hornstein, E. Vulnerability of microRNA biogenesis in FTD-ALS. Brain Res. 1647, 105-11 (2016).

154. Fourier, A. et al. Pre-analytical and analytical factors influencing Alzheimer's disease cerebrospinal fluid biomarker variability. Clin. Chim. Acta 449, 9-15 (2015).

155. Lista, S. et al. Biomarkers in Sporadic and Familial Alzheimer's Disease. J. Alzheimer's Dis. 47, 291-317 (2015).

156. Coyle-gilchrist, I. T. S. et al. Prevalence, characteristics, and survival of frontotemporal lobar degeneration syndromes. Neurology 86, 1736-1743 (2016).

157. Nilsson, C., Waldö, M. L., Nilsson, K., Santillo, A. \& Vestberg, S. Age-related incidence and family history in Frontotemporal dementia: Data from the Swedish Dementia Registry. PLoS One 9, 4-9 (2014).

158. Seelaar, H., Rohrer, J. D., Pijnenburg, Y. A. L., Fox, N. C. \& van Swieten, J. C. Clinical, genetic and pathological heterogeneity of frontotemporal dementia: a review. J. Neurol. Neurosurg. Psychiatry 82, 476-86 (2011). 